\title{
Clinical Outcomes of Early Treatment With Doxycycline for 89 High-Risk COVID-19 Patients in Long-Term Care Facilities in New York
}

\author{
Mohammud M. Alam ${ }^{1}$, Saborny Mahmud ${ }^{2}$, Mohammad M. Rahman ${ }^{3}$, JoAnn Simpson ${ }^{4}$, Sandeep \\ Aggarwal $^{5}$, Ziauddin Ahmed ${ }^{6}$ \\ 1. Department of Medicine and Infectious Diseases, Northwell University Hospital, Plainview, USA 2. Department of \\ Medicine, Johns Hopkins Bloomberg School of Public Health, Baltimore, USA 3. Department of Medicine, New York \\ University (NYU) School of Medicine, New York, USA 4. Department of Nursing, Stony Brook University, Stony Brook, \\ USA 5. Department of Nephrology, University of Pennsylvania, Philadelphia, USA 6. Department of Medicine and \\ Nephrology, Temple University, Philadelphia, USA
}

Corresponding author: Mohammud M. Alam, dr.tuku@yahoo.com

\section{Abstract}

\section{Rationale}

Due to the cluster and associated comorbidities in residents of long-term care facilities (LTCFs), COVID-19associated morbidity and mortality are significantly increased. Multiple therapeutic options, including hydroxychloroquine (HCQ) and azithromycin (AZI), were tried initially to treat moderate to severe COVID19 and high-risk patients in LTCFs, but they were abandoned due to unfavorable reports. As a less toxic option, we initiated treatment with doxycycline (DOXY) very early in the course of illness. DOXY has antiviral, cardioprotective, immunomodulatory, and anti-inflammatory properties, but the efficacy of early intervention with DOXY in high-risk COVID-19 patients in LTCFs is unknown.

\section{Objective}

The goal of this retrospective study is to describe the clinical outcomes of high-risk COVID-19 patients with moderate to severe symptoms in LTCFs after early intervention with DOXY.

\section{Design}

Case-series analysis

\section{Setting}

LTCFs in New York

\section{Participants}

Received 07/15/2020

Review began 07/25/2020

Review ended 07/26/2020

Published 08/11/2020

\section{() Copyright 2020}

Alam et al. This is an open access article distributed under the terms of the Creative Commons Attribution License CC-BY 4.0., which permits unrestricted use, distribution, and reproduction in any medium, provided the original author and source are credited.
This observational study examines 89 patients who were diagnosed with COVID-19 from March 18 to May 13, 2020.

\section{Exposure}

All patients who were diagnosed with COVID-19 received DOXY and regular standard of care within 12 hours of the onset of symptoms. Additionally, four patients received meropenem, three patients received Zosyn, two patients received linezolid, and two patients received Bactrim DS. Four patients were on chronic ventilator support. No patients received any steroids or any other antiviral or immunomodulatory agents. The majority of the patients received zinc and calcium supplements as well.

\section{Main outcomes and measures}

Assessed measures were patients' characteristics, fever, shortness of breath (SOB), cough, oxygen saturation/pulse oximetry (POX), radiologic improvements, laboratory tests, DOXY side effects, hospital transfers, and death.

\section{Results}

Eighty-nine (89) high-risk patients, who developed a sudden onset of fever, cough, SOB, and hypoxia and were diagnosed with COVID-19, were treated with DOXY (100 mg PO or intravenous (IV) for seven days) and regular standard of care. Eighty-five percent $(85 \%)$ of patients $(n=76)$ demonstrated clinical recovery that is defined as resolution of fever (average 3.7 days, Coeff $=-0.96, p=0.0001$ ), resolution of SOB (average 4.2 days), and improvement of POX: average $84 \%$ before treatment and average $95 \%$ after treatment $(84.7 \pm 7 \%$ vs. $95 \pm 2.6 \%, p=0.0001$ ). Higher pre- and post-treatment POX is associated with lower mortality (oxygen saturation $(\mathrm{Spo} 2)$ vs. Death, Coeff $=-0.01, \mathrm{p}=0.023$; post-Spo2 vs. Death, Coeff $=-0.05, \mathrm{p}=0.0002$ ). Within 
10 days of symptom onset, $3 \%$ of patients $(n=3)$ were transferred to hospital due to clinical deterioration and $11 \%$ of patients $(n=10)$ died. The result was followed for 30 days from the onset of symptoms in each patient.

\section{Conclusion}

Early treatment with DOXY for high-risk patients with moderate to severe COVID-19 infections in nonhospital settings, such as LTCFs, is associated with early clinical recovery, decreased hospitalization, and decreased mortality.

Categories: Infectious Disease

Keywords: covid-19, doxycycline, treatment, long-term care facility

\section{Introduction}

The COVID-19 pandemic placed an unprecedented and overwhelming burden on the U.S. healthcare system. Since the first case of COVID-19 in the U.S., there have been over two million COVID-19 cases and 122,000 deaths as of June 25, 2020 [1]. One-third of these deaths are nursing home residents or workers [2]. Data from small studies conducted in China [3] and France [4] present the clinical outcomes of COVID-19 patients who were treated with hydroxychloroquine (HCQ) and/or azithromycin (AZI). However, since HCQ and AZI are both cardiotoxic, there are concerns about the development of arrhythmia in patients treated with these drugs [5]. With limited treatment options, our group first treated high-risk COVID-19 patients in long-term care facilities (LTCFs) with doxycycline (DOXY) and HCQ [6]. DOXY has anti-inflammatory [7], immunomodulating [8], cardioprotective [9-10], and antiviral [11] activities. Subsequently, other studies noted the harmful side effects and mortality associated with HCQ administration [12].

Based on current evidence and reference articles that detail COVID-19 pathophysiology and DOXY's mechanisms of action, DOXY may be an effective drug in the treatment of COVID-19. As a result, we began treating high-risk patients with only DOXY and supportive care. The efficacy of early DOXY intervention in high-risk COVID-19 patients in LTCFs is unknown. In this observational study, we collected data retrospectively, and we are presenting our clinical observations and outcomes of DOXY for high-risk patients with moderate to severe COVID-19 infections in LTCFs.

\section{Materials And Methods}

This case series assessed the clinical outcomes of eighty-nine (89) high-risk patients in LTCFs who developed a sudden onset of fever, cough, shortness of breath (SOB), and hypoxia between March 18 and May 13, 2020. High-risk patients were defined as patients who had at least one comorbidity such as hypertension (HTN), diabetes, coronary artery disease (CAD), congestive heart failure (CHF), chronic obstructive pulmonary disorder (COPD), obesity, or ventilator-dependency. These patients were diagnosed with COVID-19 and they were treated with an early course of DOXY (100 mg PO or IV for seven days) and regular standard of care. Since all patients were closely monitored by healthcare workers, COVID-19 symptoms were immediately noticed; DOXY was started within 12 hours and RT-PCR COVID-19 swab was collected. Assessed measures were patients' characteristics, fever, SOB, cough, oxygen saturation/pulse oximetry (POX), radiologic improvements, laboratory tests, DOXY side effects, hospital transfers, and death. Clinical recovery was defined as a resolution of fever, resolution of SOB, and improvement of POX ( $\geqslant 93 \%)$. Followup chest X-rays (CXR) were ordered if clinically indicated.

The statistical analysis was done using Microsoft Excel 2016 (Microsoft Corporation, Redmond, Washington) with statistical application packages and IBM SPSS v23 (IBM Corp., Armonk, NY). The two-tailed T-test was used for between-group intervals in parametric data (e.g. POX). Binary logistic analysis and regression were used to assess the association of risk factors with the outcome. The patients' outcomes were recorded 30 days after symptoms onset.

All patients or patients' families gave informed consent before DOXY treatment was started, and oversight medical boards and corporate clinical services approved this observational study.

\section{Results}

Table 1 summarizes the characteristics, clinical features, lab and radiology results, outcomes, and side effects of DOXY of all 89 patients who were started with early DOXY treatment. The median age is 78 years, and the range is $43-101$ years. One hundred percent (100\%) of patients $(n=89)$ tested positive for COVID-19 through reverse transcription-polymerase chain reaction (RT-PCR) and $85 \%$ of patients (n=76) showed clinical recovery. Eleven percent $(11 \%)$ of patients died $(n=10)$ and $3 \%$ of patients $(n=3)$ were transferred to hospitals due to clinical deterioration. 


\section{Cureus}

\begin{tabular}{|c|c|c|}
\hline Median Age, Years (Range) & $78(43-101)$ & \\
\hline Male & 50 & $56 \%$ \\
\hline Female & 39 & $44 \%$ \\
\hline Clinically Recovered ${ }^{*}$ & 76 & $85 \%$ \\
\hline Transferred to Hospital & 3 & $3 \%$ \\
\hline Death & 10 & $11 \%$ \\
\hline Hypertension (HTN) & 62 & $70 \%$ \\
\hline Diabetes & 30 & $34 \%$ \\
\hline Coronary Artery Disease (CAD) & 41 & $46 \%$ \\
\hline Congestive Heart Failure (CHF) & 23 & $26 \%$ \\
\hline Chronic Obstructive Pulmonary Disease (COPD) & 31 & $35 \%$ \\
\hline Obesity & 37 & $42 \%$ \\
\hline Ventilator Dependency ${ }^{\star \star}$ & 4 & $4 \%$ \\
\hline Fever & 87 & $98 \%$ \\
\hline Shortness of Breath (SOB) & 85 & $96 \%$ \\
\hline Cough & 83 & $93 \%$ \\
\hline Malaise/Weakness & 71 & $80 \%$ \\
\hline Altered Mental Status & 56 & $63 \%$ \\
\hline Diarrhea & 31 & $35 \%$ \\
\hline Chest Pain & 27 & $30 \%$ \\
\hline Tested COVID-19 Positive via RT-PCR & 89 & $100 \%$ \\
\hline High BUN & 50 & $56 \%$ \\
\hline High CR & 46 & $52 \%$ \\
\hline High LFT & 33 & $37 \%$ \\
\hline High Regular CRP & 65 & $73 \%$ \\
\hline High Ferritin & 63 & $71 \%$ \\
\hline High LDH & 34 & $38 \%$ \\
\hline High D-Dimer & 57 & $64 \%$ \\
\hline High Troponin & 40 & $45 \%$ \\
\hline High Procalcitonin & 34 & $38 \%$ \\
\hline Chest X-Ray (CXR) with Pneumonia & 85 & $96 \%$ \\
\hline Chest X-Ray (CXR) Improved & 39 & $44 \%$ \\
\hline Chest X-ray (CXR) Not Improved & 41 & $46 \%$ \\
\hline Chest X-Ray (CXR) Unavailable ${ }^{\star \star \star ~}$ & 9 & $10 \%$ \\
\hline Nausea, Vomiting, and Abdominal Discomfort & 6 & $7 \%$ \\
\hline No Side Effects & 83 & $93 \%$ \\
\hline Other Antibiotics & 11 & $12 \%$ \\
\hline
\end{tabular}

TABLE 1: Characteristics, clinical outcomes, clinical features, lab and radiology results, side effects of doxycycline, and other antibiotics of all 89 high-risk COVID-19 patients who started 


\section{Cureus}

\section{seven-day doxycycline treatment in long-term care facilities}

*Clinical recovery defined by resolution of fever (3.7 days), resolution of SOB (4.2 days), and average POX improvement $95 \%$

**Four percent $(n=4)$ of ventilator-dependent patients recovered and the ventilator setting returned to baseline.

${ }^{\star \star \star}$ Chest X-ray not ordered, not required, or pending.

BUN: blood urea nitrogen; CRP: C-reactive protein; LFT: liver function test; LDH: lactate dehydrogenase; POX: pulse oximetry

Table 2 summarizes the characteristics, clinical features, lab and radiology results, outcomes, and side effects of DOXY of the remaining 76 patients who successfully completed the seven-day DOXY treatment. One hundred percent (100\%) of patients demonstrated clinical improvement. After DOXY treatment started, resolution of fever and SOB occurred at an average of 3.7 days and 4.2 days, respectively. Average POX before and after treatment was $84 \%$ and $95 \%$, respectively.

\begin{tabular}{|c|c|c|}
\hline Assessed Measures & LTCF Residents ( $n=76$ ) & Percentage \\
\hline Median Age, Years (Range) & $79(43-101)$ & \\
\hline Male & 42 & $55 \%$ \\
\hline Female & 34 & $45 \%$ \\
\hline Clinically Recovered ${ }^{*}$ & 76 & $100 \%$ \\
\hline Hypertension (HTN) & 52 & $68 \%$ \\
\hline Diabetes & 25 & $33 \%$ \\
\hline Coronary Artery Disease (CAD) & 35 & $46 \%$ \\
\hline Congestive Heart Failure (CHF) & 19 & $25 \%$ \\
\hline Chronic Obstructive Pulmonary Disease (COPD) & 26 & $34 \%$ \\
\hline Obesity & 33 & $43 \%$ \\
\hline Ventilator Dependency $y^{\star \star}$ & 4 & $4 \%$ \\
\hline Fever & 74 & $97 \%$ \\
\hline Shortness of Breath (SOB) & 72 & $95 \%$ \\
\hline Cough & 71 & $93 \%$ \\
\hline Chest Pain & 21 & $28 \%$ \\
\hline Malaise/Weakness & 62 & $82 \%$ \\
\hline Altered Mental Status & 45 & $59 \%$ \\
\hline Diarrhea & 25 & $33 \%$ \\
\hline COVID-19 Positive & 76 & $100 \%$ \\
\hline High BUN & 39 & $51 \%$ \\
\hline High CR & 39 & $51 \%$ \\
\hline High LFT & 26 & $34 \%$ \\
\hline High Regular CRP & 53 & $70 \%$ \\
\hline High Ferritin & 53 & $70 \%$ \\
\hline High LDH & 27 & $36 \%$ \\
\hline High D-Dimer & 48 & $63 \%$ \\
\hline High Iroponin & 32 & $42 \%$ \\
\hline High Procalcitonin & 28 & 37 \\
\hline
\end{tabular}




\section{Cureus}

\begin{tabular}{|c|c|c|}
\hline Individuals with Pulse Oximetry (POX) Between $60 \%$ and $69 \%$ Before Treatment & 4 & $5 \%$ \\
\hline Individuals with Pulse Oximetry (POX) Between $70 \%$ and $80 \%$ Before Treatment & 18 & $20 \%$ \\
\hline Individuals with Pulse Oximetry (POX) Between $81 \%$ and $89 \%$ Before Treatment & 42 & $47 \%$ \\
\hline Individuals with Pulse Oximetry (POX) Between $90 \%$ and $92 \%$ Before Treatment & 25 & $28 \%$ \\
\hline Average Pulse Oximetry (POX) After Treatment & 76 & $95 \%$ \\
\hline Chest X-Ray (CXR) with Pneumonia & 72 & $95 \%$ \\
\hline Chest X-Ray (CXR) Improved & 36 & $47 \%$ \\
\hline Chest X-Ray (CXR) Not Improved & 31 & $41 \%$ \\
\hline Chest X-Ray (CXR) Unavailable ${ }^{\star \star \star}$ & 9 & $12 \%$ \\
\hline Nausea, Vomiting and Abdominal Discomfort & 6 & $8 \%$ \\
\hline No Side Effects & 70 & $92 \%$ \\
\hline Other Antibiotics & 6 & $8 \%$ \\
\hline
\end{tabular}

\section{TABLE 2: Characteristics, clinical outcomes, clinical features, lab and radiology results, side effects of doxycycline, and other antibiotics of 76 high-risk COVID-19 patients who completed seven-day doxycycline treatment in long-term care facilities}

This table includes all patients who completed a full course (7 days) of DOXY therapy and excludes any patients who died and who were transferred to hospital and did not complete the seven-day course.

${ }^{\star}$ Clinical recovery defined by resolution of fever (3.7 days), resolution of SOB (4.2 days), and average POX improvement $95 \%$ after treatment.

${ }^{\star \star}$ Four percent $(n=4)$ ventilator-dependent patients recovered and the ventilator setting returned to baseline.

${ }^{\star \star \star}$ Chest X-ray either not ordered, or not required or pending.

BUN: blood urea nitrogen; CRP: C-reactive protein; LFT: liver function test; LDH: lactate dehydrogenase; POX: pulse oximetry

There was a statistically significant difference in POX before (pre-POX) and after (post-POX) treatment ( $\mathrm{n}=$ $89,84.7 \pm 7 \%$ vs. $95 \pm 2.6 \%, p=0.0001$ ). In the multivariate analysis model, the resolution of fever was associated with a reduction in mortality (Coeff $=-0.96, \mathrm{p}=0.0001$ ). Higher pre-POX and post-POX were also associated with decreased mortality; lower pre- and post-treatment POX is associated with increased mortality (pre-oxygen saturation $(\mathrm{Spo2})$ vs Death, Coeff $=-0.01, \mathrm{p}=0.023$; Post-spo2 vs Death, Coeff $=-0.05$, $\mathrm{p}=0.0002$ ). Other variables, such as initial CXR with pneumonia, BUN, creatinine, liver enzyme levels, CRP, ferritin, LDH, D-Dimer, troponin, and procalcitonin, were not associated with mortality. Overall mortality at 30 days in our cohort was $11 \%(\mathrm{n}=10)$. Clinical improvement was noted in 76 patients. Additionally, $3 \%$ $(n=3)$ required transfer to a hospital while the rest of the patients were treated in the nursing home facility.

\section{Discussion}

COVID-19 is a positive-sense, single-stranded ribonucleic acid (RNA) virus [13], with a lipophilic outer shell that allows the pathogen to easily infiltrate lung tissue [14]. The coronavirus is believed to recognize and bind to cluster of differentiation 26 (CD26)/dipeptidyl peptidase-4 (DPP4) markers expressed on the cell surface, serving as an entry point for viral invasion [15]. COVID-19 infects the upper and lower respiratory tract, causing the release of pro-inflammatory cytokines such as interleukin $1 \beta$ (IL-1 $1 \beta$ ), tumor necrosis factor (TNF), and IL-6 [16]. COVID-19 induces mast cell proliferation within the respiratory submucosa [17] and activates the NF-kB pathway [18], further increasing the inflammatory response. As a result, individuals with COVID-19 present with fever, cough, and SOB. Severe infection can progress to acute respiratory failure and vascular thrombosis. COVID-19 is additionally correlated with acute cardiac injury and increased troponin I levels [19].

DOXY, an analog of tetracycline, is lipophilic and can also easily penetrate the lung epithelium [20]. Chemically modified tetracyclines (CMTs) can also induce apoptosis of mast cells [21]. The drug downregulates inflammatory markers demonstrated to have a role in COVID-19 pathophysiology. Evidence shows that DOXY downregulates the expression of DPP4 through the inhibition of the NF-kB pathway [22], impeding the virus's ability to enter cells and consequently decreasing the viral load. Along with its antiinflammatory and immunomodulatory properties, DOXY is considered to be cardioprotective. During 
reperfusion after myocardial injury, matrix metalloproteinases-2 (MMP-2) are released. DOXY's inhibition of the MMP-2 pathway rescues left ventricular function [23]. As a result, in patients with acute ST-segment elevation myocardial infarct (STEMI) and left ventricular dysfunction, DOXY reduces adverse left ventricular remodeling [24]. DOXY's cardioprotective characteristics may improve clinical recovery in COVID-19 patients with acute myocardial injury.

DOXY is also thought to have antiviral activity. In-vitro assays of cultured cells inoculated with the dengue virus suggest that DOXY inhibits the virus's serine protease, disrupting viral replication, and viral entry into cells [25]. Another study demonstrates that when cells infected with murine retrovirus were treated with DOXY, there was a $70 \%$ decrease in retroviral load [26]. Tetracyclines can potentially treat other viral infections as well, such as viral encephalitis, West Nile virus, and human immunodeficiency virus (HIV) [27]. Further research reveals that elevated levels of intracellular zinc inhibit viral replication of COVID-19 [28] and that DOXY facilitates this inhibition by acting as a zinc ionophore, transporting, and increasing intracellular concentrations of zinc. Recently, a study demonstrated that in-vitro, DOXY itself has antiviral activity against COVID-19 at a concentration of $5.6 \mu \mathrm{M}$ [29].

DOXY has anti-inflammatory, immunomodulatory, cardioprotective, and antiviral properties. No observational study has yet been done using an early regimen of DOXY to treat moderate to severe high-risk COVID-19 patients in an LTCF. This case series demonstrates that early DOXY treatment in a non-hospital environment has good clinical recovery $(85 \%, n=76)$, minimized hospital transfers $(3 \%, n=3)$, and reduced death $(11 \%, n=10)$.

A limit to this study is that there was no control group. We thus compared the data from this population to that of a similar reference patient population in an LTCF in Washington. This study performed an epidemiological investigation regarding 129 confirmed cases of COVID-19. Of the 129 confirmed cases, $63 \%$ $(\mathrm{n}=81)$ were LTCF residents (median age $=81$ years, range $=54-100$ years), $26 \%(\mathrm{n}=34)$ were healthcare workers (median age $=42.5$ years, range $=22-79$ years, and $11 \%(n=14)$ were visitors (median age $=62.5$ years, range $=52-88$ years). Fifty-six point eight $(56.8 \%)$ LTCF residents, $35.7 \%$ visitors, and $5.9 \%$ of healthcare personnel were hospitalized. Comorbidities among LTCF residents included HTN (69.1\%), cardiac disease (56.8\%), diabetes (37\%), obesity (33\%), and pulmonary disease (32.1\%). Of the total population, $65.1 \%(\mathrm{n}=84)$ were women. While no deaths occurred among staff members, $27.2 \%$ of LTCF residents and $7.1 \%$ of visitors died [30]. Comparing this study's data to our data using naive indirect comparison, early DOXY treatment may reduce hospital transfers and decrease mortality.

\section{Conclusions}

In this cohort of high-risk LTCF residents with moderate to severe COVID-19 infections who were treated with an early course of DOXY, improvement in oxygen saturation and resolution of fever were factors associated with lower mortality. The median time of all deaths in these high-risk patients was within the first 10 days in the course of illness. In the majority of our cohort, early initiation of DOXY treatment was associated with improved clinical outcomes, decreased hospitalizations, and decreased mortality. Larger randomized control trials are required to study the effect of DOXY treatment in moderate to severe COVID19 infections.

\section{Additional Information \\ Disclosures}

Human subjects: Consent was obtained by all participants in this study. Animal subjects: All authors have confirmed that this study did not involve animal subjects or tissue. Conflicts of interest: In compliance with the ICMJE uniform disclosure form, all authors declare the following: Payment/services info: All authors have declared that no financial support was received from any organization for the submitted work. Financial relationships: All authors have declared that they have no financial relationships at present or within the previous three years with any organizations that might have an interest in the submitted work. Other relationships: All authors have declared that there are no other relationships or activities that could appear to have influenced the submitted work.

\section{References}

1. New cases of COVID-19 In world countries . (2020). Accessed: June 25, 2020: https://coronavirus.jhu.edu/data/new-cases.

2. New York Times. One-third of all U.S coronavirus deaths are nursing home residents or workers . (2020). Accessed: May 9, 2020: https://www.nytimes.com/interactive/2020/05/09/us/coronavirus-cases-nursinghomes-us.html.

3. Chen Jun, Liu Danping, Liu Li, et al.: A pilot study of hydroxychloroquine in treatment of patients with moderate COVID-19 [Article in Chinese]. Zhejiang Da Xue Xue Bao Yi Xue Ba. 2020, 49:215-219. 10.3785/j.issn.1008-9292.2020.03.03

4. Philippe G, Lagier J-C, Parola P, et al.: Hydroxychloroquine and azithromycin as a treatment of COVID- 19: results of an open-label non-randomized clinical trial. Int J Antimicrob Agents. 2020, 56:105949.

10.1016/j.ijantimicag.2020.105949 
5. Potential harms of chloroquine, hydroxychloroquine and azithromycin for treating COVID-19 . (2020). https://www.sciencedaily.com/releases/2020/04/200408125521.htm.

6. Ahmad I, Alam M, Saadi R, Mahmud S, Saadi E: Doxycycline and hydroxychloroquine as treatment of highrisk COVID-19 patients: experience from case series of 54 patients in long term care facilities [Preprint]. medRxiv. 2020, 10.1101/2020.05.18.20066902

7. McKeage K, Deeks ED: Doxycycline $40 \mathrm{mg}$ capsule (30 mg immediate-release/10 mg delayed-release beads): anti-inflammatory dose in rosacea. Am J Clin Dermatol. 2010, 11:217-222. 10.2165/11204850-00000000000000

8. Mishra A, Bhattacharya P, Paul S, Paul R, Swarnakar S: An alternative therapy for idiopathic pulmonary fibrosis by doxycycline through matrix metalloproteinase inhibition. Lung India. 2011, 28:174-179. 10.4103/0970-2113.83972

9. Cerisano G, Buonamici P, Valenti R, et al.: Early short-term doxycycline therapy in patients with acute myocardial infarction and left ventricular dysfunction to prevent the ominous progression to adverse remodelling: the TIPTOP trial. Eur Heart J. 2014, 35:184-191. 10.1093/eurheartj/eht420

10. Villarreal FJ, Griffin M, Omens J, Dillmann W, Nguyen J, Covell J: Early short-term treatment with doxycycline modulates postinfarction left ventricular remodeling. Circulation. 2003, 108:1487-1492. 10.1161/01.CIR.0000089090.05757.34

11. te Velthuis AJ, van den Worm SH, Sims AC, Baric RS, Snijder EJ, van Hemert MJ: Zn(2+) inhibits coronavirus and arterivirus RNA polymerase activity in vitro and zinc ionophores block the replication of these in cell culture. PLoS Pathog. 2010, 4:6. 10.1371/journal.ppat.1001176

12. Magagnoli J, Narendran S, Pereira F, Cummings TH, Hardin JW, Sutton SS, Ambati J: Outcomes of Hydroxychloroquine Usage in United States Veterans Hospitalized with COVID-19. Clin Adv. 2020, [Epub ahead of print]:10.1016/j.medj.2020.06.001

13. DediegoML, Nieto-Torres, Regla-Nava JA, et al.: Inhibition of NF-kB-mediated inflammation in severe acute respiratory syndrome coronavirus infected mice increases survival. J Virol. 2014, 88:913-924. 10.1128/JVI.02576-13

14. Sargiacomo C, Sotgia F, Lisanti MP: COVID-19 and chronological aging: senolytics and other anti-aging drugs for the treatment or prevention of coronavirus infection?. Aging (Albany NY). 2020, 12:6511-6517. 10.18632/aging.103001

15. Choi B, Lee S, Kim SM, et al.: Dipeptidyl peptidase-4 induces aortic valve calcification by inhibiting insulinlike growth factor-1 signaling in valvular interstitial cells. Circulation. 2017, 16:1935-1950. 10.1161/CIRCULATIONAHA.116.024270

16. Kritas SK, Ronconi G, Caraffa A, Gallenga CE, Ross R, Conti P: Mast cells contribute to coronavirus-induced inflammation: new anti-inflammatory strategy. J Biol Homeost Agents. 2020, 34:[Epub ahead of print]. 10.23812/20-Editorial-Kritas

17. Sodhi M, Etminan M: Therapeutic potential for tetracycline in the treatment of COVID-19 . Pharmacotherapy. 2020, 40:487-488. 10.1002/phar.2395

18. Kumar N, Xin ZT, Liang Y, Ly H, Liang Y: NF-kappaB signaling differentially regulates influenza virus RNA synthesis. J Virol. 2008, 92:9880-9889. 10.1128/JVI.00909-08

19. Shi S, Qin M, Shen B, et al.: Association of cardiac injury with mortality in hospitalized patients with COVID-19 in Wuhan, China. JAMA Cardiol. 2020, 5:802-810. 10.1001/jamacardio.2020.0950

20. Griffin MO, Fricovsky E, Ceballos G, Villarreal F: Tetracyclines: a pleitropic family of compounds with promising therapeutic properties. Review of the literature. Am J Physiol Cell Physiol. 2010, 299:C539-548. 10.1152/ajpcell.00047.2010

21. Sandler C, Nurmi K, Lindstedt KA, Sorsa T, Golub LM, Kovanen PT, Eklund KK: Chemically modified tetracyclines induce apoptosis in cultured mast cells. Int Immunopharmacol. 2005, 5:1611-1621. 10.1016/j.intimp.2005.04.013

22. Choi B, Lee S, Kim SM, et al.: Dipeptidyl peptidase-4 induces aortic valve calcification by inhibiting insulinlike growth factor-1 signaling in valvular interstitial cells. Circulation. 2017, 16:1935-1950. 10.1161/CIRCULATIONAHA.116.024270

23. Villarreal FJ, Griffin M, Omens J, Dillmann W, Nguyen J, Covell J: Early short-term treatment with doxycycline modulates postinfarction left ventricular remodeling. Circulation. 2003, 108:1487-1492. 10.1161/01.CIR.0000089090.05757.34

24. Cerisano G, Buonamici P, Valenti R, et al.: Early short-term doxycycline therapy in patients with acute myocardial infarction and left ventricular dysfunction to prevent the ominous progression to adverse remodelling: the TIPTOP trial. Eur Heart J. 2014, 35:184-191. 10.1093/eurheartj/eht420

25. Rothan HA, Mohamed Z, Paydar M, Rahman NA, Yusof R: Inhibitory effect of doxycycline against dengue virus replication in vitro. Arch Virol. 2014, 159:711-718. 10.1007/s00705-013-1880-7

26. Sturtz FG: AntiMurine retroviral effect of doxycycline. Methods Find Exp Clin Pharmacol. 1998, 20:643-647. 10.1358/mf.1998.20.8.487489

27. Dutta K, Basu A: Use of minocycline in viral infections . Indian J Res. 2011, 133:467-470.

28. Saper RB, Rash R: Zinc: an essential micronutrient. Am Fam Physician. 2009, 79:799-772.

29. In vitro antiviral activity of doxycycline against SARS-CoV-2 . (2020). http://www.mediterraneeinfection.com/in-vitro-antiviral-activity-of-doxycycline-against-sars-cov-2/.

30. McMichael TM, Clark S, Pogosjans S, et al.: COVID-19 in a long-term care facility - King County, Washington, February 27-March 9. MMWR Morb Mortal Wkly Rep. 2020, 69:339-342. 10.15585/mmwr.mm6912e1 